\title{
Optimization on reagent-loading manner for modular clinical chemistry analyzer series: simulations and verifications
}

\author{
https://doi.org/10.1515/labmed-2020-0075 \\ Received April 15, 2020; accepted July 31, 2020; published online \\ September 7, 2020
}

\begin{abstract}
Objectives: The pre- and post-analytical processes have been discussed both in total laboratory system (TLA) and modular automation (MA). The analytical process, especially reagent-related factors influences on the integrated clinical chemistry analyzer, demonstrates a significant effect on clinical chemistry analyzer. Modular analyzer reagent-loading mode influences two mainly factors, testing turnaround time (tTAT) and the cost. Furthermore, how to definite the different reagent loading manners and verify the best reagent loading manner is big challenge.

Methods: We focus on tTAT, and study how the reagentrelated factors effect TAT by simulations and verifications. Parameters were simulated by cobas 8000 workflow simulator for reagent-loading manner with at least three positions (Pattern 1), the module-parallel reagent-loading manner (Pattern 2) and the single-position loading mode (Pattern 3). Results: tTAT, reagent on-line time, quality control (QC) cost and performance verification times all declined by $43 \%$. Tuesday effect solved the repetitive problem for verification. Pattern 2 shows optimal performance in Tuesday effect-based verification.

Conclusions: The optimization of reagent-loading manner saved much workforce, and reduced the QC cost.
\end{abstract}

Keywords: modular analyzer series; reagent-loading manner simulation; reagent-loading manner verification; testing turnaround time.

\section{Introduction}

Clinical chemistry results take up a large proportion in modern healthcare systems. It helps the clinical decisions

*Corresponding author: Liang Ming, Department of Clinical Laboratory Medicine, The First Affiliated Hospital of Zhengzhou University, Zhengzhou, P.R. China, E-mail: mingliang3072@163.com Mingyang Wang, Department of Clinical Laboratory Medicine, The First Affiliated Hospital of Zhengzhou University, Zhengzhou, P.R. China and bridges the gap between the physicians and patients. For a better evaluation, the total clinical process has been separated into the pre-analytical, analytical and post-analytical phase. Meanwhile, turnaround time (TAT) has also been defined as a quality indicator in laboratory medicine clinical process, and it can be further subdivided into the pre-analytical related TAT and analytical related TAT depending on points in the total testing process [1]. Preanalytical related TAT includes the points from the moment that the clinicians confirm the test panel to the moment that the sample is ready for test. Clinical analytical related TAT is calculated from the moment when the plasma is registered by analyzer to the point when the test results are verified by technicians. The results can be effect by instrument setups, analytical methodologies, reagent-related factors, and testing process optimization, such as labor, reagent, test panel, laboratory management and energy cost [2].

For total laboratory system (TLA) and modular automation (MA), the pre- and post-analytical processes have been discussed more than the analytical process [3], which is based on the clinical chemistry analyzer and should also draw our attention. As critical component of clinical chemistry lab, clinical chemistry analyzer has effect on two important factors, the clinical analytical related TAT and the cost $[4,5]$, which mainly determine the operating cost of laboratories and quality of laboratory services. When the specimen amount of is constant, the reduction of TAT relies on the improvement of the equipments. And then, the less TAT one needs, the more satisfaction one gets [6-8].Clinical chemistry analyzer reagent-loading manner impacts on TAT is deficiency in the analytical process. Here, we research clinical chemistry analyzer-related analytical TAT, and study how the reagent-related factors effect TAT. Testing TAT (tTAT) covers the period between the moment of first rack being sent into the machine and the moment of last test results in the rack being sent to the laboratory information system (LIS) [3].

For analyzers and modules in MA, LIS can only display the time points when the tube barcodes are read by the aliquoter and when the test results are sent. Nevertheless, the time points of samples loading, 
barcodes registration, tests carriers or rack unloading and test results sending among tTAT in the workflow are unknown. What's more, how to analyze and optimize tTAT is still a big challenge even if one can calculate these time points. Data simulation is a useful tool to handle the problem [9].

The First Affiliated Hospital of Zhengzhou University is a tertiary general hospital, consisting of three zones, Heyi zone, East branch zone and Huiji zone. The Department of Clinical Medicine in the First Affiliated Hospital of Zhengzhou University has three divisions separated in three zones. Divisions in Heyi zone and East branch zone run core laboratories, which provide services of clinical chemistry, immunology, microbiology, hematology and molecular diagnostics. From 2007 to 2012, clinical chemistry laboratory in Heyi zone imported five modular analyzer series successively, which consist two aliquoters and 16 chemistry modules in total. Therefore, chemistry modules were the main force in our lab. For a long time, the high cost and resource-consuming reagents loading for every analyzer was carried out after clinical tests completed. In order to solve this problem, we designed a new reagent-loading manner for the clinical analyzer based on simulations. cobas 8000 workflow simulator works well based on the running data analysis of the clinical chemistry analyzer before. Furthermore, the design was verified by experiments and it shows that the new reagent-loading manner reduced the cost and shortened the tTAT. These studies here will be very attractive on the aspects of shortening sample TAT and promoting analytical quality control (QC) in clinical chemistry laboratories.

\section{Materials and methods}

\section{Laboratory setup}

In this study, we used the modular analyzer series, which includes p610 aliquoter and five analyzers. These analytical systems were used to test the reagent-loading mode effects on analytical units in MA. The p610 opens lids of the sample tubes, reads the racks, sample identifications and sorts tubes within the rack [10]. We chose one modular analyzer series (Roche Diagnostic, cobas 8000 c701 X 2 c502 X 1 e602 X 1) for better studying the reagent-loading manner effects on tTAT. One cobas 8000 analyzer contains two identical c701 modules, which comprise four disks and 140 reagent bins in total.

\section{Data analysis and simulation verifications}

Our project was divided into three processes. Firstly, we analyzed current reagent setup (Pattern 1) of the analyzer series, especially for the reagent-loading parameters. Secondly, simulations for reagentloading optimization, aiming to improve the efficiency and cost reduction, proceed with cobas 8000 workflow simulator. cobas 8000 workflow simulator was used to retrospective and prospective analysis, simulation for one configuration of the cobas 8000 modular analyzer and gives answers to TAT. cobas 8000 workflow simulator includes four functionalities: overview interface, simulation interface, view output interface and setup interface. The analyzer data was the actual laboratory data and collected as CSV format from data manager, which contain sample ID, tests, sample ID reading time and sample unloading time. Then, these data was input into the simulator for configuration defining (overview interface). Modules type choices and mapping, data mapping, tests matching and distribution, mapping of analytes were operated in simulation interface. Analytical tests in one modular are corresponding to reagents' types. This step, containing tests matching and distribution, was important in this work. Because it involves reagent situation in the modules' reagent disks and eventually, decides reagent-loading manner. It is worth noting that user-defined reagents were replaced by similar vendor-supplied reagents in the simulation process. Once these steps above have done, cobas 8000 workflow simulator creates a named project. Then, simulation results for one project were calculated in the view output interface. It mainly includes the TTAT- 1 and tTAT-2, which could be used for better comparisons with different projects. Pattern 2 and Pattern 3 were theoretical reagent-loading design schemes. TATs in these two theoretical reagent-loading manners were simulated based on Pattern 1. Parameters and differences were derived by cobas 8000 workflow simulator. Finally, we chose Pattern 2 for the simulation verification and it works best until now.

\section{Results}

\section{Simulation foundation - the Tuesday effect}

Patients in the First Hospital of Zhengzhou University have a rhythm (Figure 1), which we call the Tuesday effect and termed as specimen summit. People always choose to make an appointment with the doctor through the outpatient service on Monday. Then, the doctors' advices were given to the patients and some of them would be hospitalized depending on the diseases and became inpatients. On the Tuesday's morning, routine tests would be conducted aiming to evaluate the present patients' conditions. These tests were clinical chemistry related assays and represented by liver and renal function assays, routine chemistry panel, blood glucose, myocardial enzymes and lipids profile listed in Table 1. Due to the Tuesday effect, $3,730 \pm 100$ clinical chemistry specimens would be tested and they had similar test profile on every Tuesday. Tuesday blood specimens account for $19.61 \pm 0.44 \%$ of total specimens in one week. The analytical unit studied here was one of the five cobas 8000 analytical analyzer series and the serums were sorted equally by p610 aliquoter. It is impossible to use all the same samples in one Tuesday to 


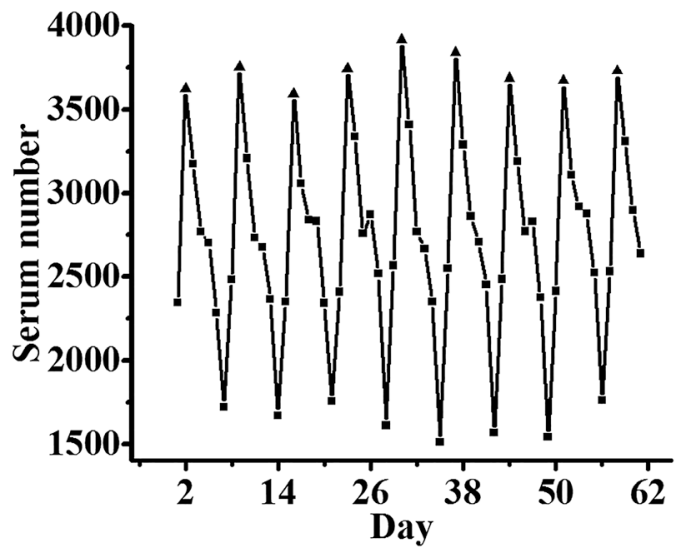

Figure 1: The tendency chart and the Tuesday effect of clinical chemistry serums in our clinical chemistry laboratory within 62 days. Our test tube number was between 300 and 800 (almost 5,00015,000 clinical chemistry tests) within a week. Black triangles represent the serum numbers on Tuesday and black squares were the other day. The TTAT and reagent-loading pattern before optimization.

repeated test or verified in another day for differentiating the reagent-loading modes. But, this rhythm in clinical serum samples supports that our study can get almost the same serum test conditions in different Tuesdays. Ultimately, we could verify the cobas 8000 workflow simulator result at the same condition to evaluate the reagentloading manner.

Pre-analytical related TAT includes centrifuging, charging, racking, transporting, serum tube distribution and test assignment [11]. Analytical related tTAT was effect by instruments, analytical methodologies, and reagentrelated factors [12]. Here, we focused on analytical related tTAT, and studied the reagent-related factors effects on TAT. tTAT covered the average TAT between the first rack being sent into analyzer and the last test results in the rack being sent to the LIS. For detail, tTAT contained two statistical patterns, tTAT-1 and tTAT-2 (Figure 2). tTAT-1 represents the average time from the sample loading or registration to rack unloading. TTAT-2 covered the time points from rack unloading to last result sending in the rack.

Due to the analytical requests of the in- and outpatients and the Tuesday effect, we incessantly imported five modular analytical series during 2007 and 2012. We chose one series as research model, which contains one ISE 900 electrolytes module, two c701 and one c502 clinical chemistry modules, and one e602 immunechemistry module. Blood electrolytes, clinical chemistry and immunechemistry of requested tests account for 9, 90 and $1 \%$ (Supplemental Figure 1), respectively. Thus, electrolytes
Table 1: Liver function panel, renal function panel, lipids profile, myocardial enzymes, blood glucose and others tests were belong to clinical chemistry tests and tested in c701 or c502 clinical chemistry modules.

\begin{tabular}{ll}
\hline Panels & Tests \\
\hline Liver function panel & $\begin{array}{c}\text { ALT, AST, GGT, ALP, TP, ALB, TBIL, BILD, TBA, } \\
\text { PA, CHE, AFU, CP, ASTm, 5'NT, ADA }\end{array}$ \\
$\begin{array}{l}\text { Renal function panel } \\
\text { Lipids profile }\end{array}$ & $\begin{array}{l}\text { Trea, Crea, UA, } \beta \text {-MG, Cys C, } \alpha-M G \\
\text { hsCRP, ACE, SOD }\end{array}$ \\
$\begin{array}{l}\text { Myocardial enzymes } \\
\text { Myocardial injure } \\
\text { markers }\end{array}$ & $\begin{array}{l}\text { AST, LDH, CK, HBDH, CK-MB, LDH-1, IMA } \\
\text { Electrolyte panel }\end{array}$ \\
$\begin{array}{l}\text { Blood glucose } \\
\text { Immunechemistry } \\
\text { tests }\end{array}$ & $\begin{array}{l}\text { KL Na, Cl } \\
\text { Others tests }\end{array}$ \\
\hline
\end{tabular}

The full names for the tests above are listed in Supplemental Table 1.

and clinical chemistry requested tests were the main factors for tTAT. Due to the rapid test for electrolytes, only the clinical chemistry modules represented by c701 units were our research focus.

The c701 unit is composed of two parts, disk A and disk $\mathrm{B}$, each of which has 35 reagent bins. The 502 module contains 60 reagent bins. Our analyzer series has two chemistry units and 140 reagent packs in total. For one series, we always employ Pattern 1 which ensured that at least three reagent kits of one test could be used at the same time for two c701 (Supplemental Table 2). This mode remains effective in our lab, and ensures that every cobas 8000 achieves the shortest tTAT and comparably uniform species distribution. For this reagent-loading mode, average tTAT-1 and tTAT were listed in Table 2 (Pattern 1).

We took it for granted that Pattern 1 can not only reduce reagent loading time but also save the labor. In fact, Pattern 1 was laborious during use due to loading reagents on the basis of four disks and 140 reagent bins for almost 40 clinical chemistry tests. Considering this situation, we established a standard operation process, and ran from 8:00 a.m. to 5:30 p.m. We conducted all the tests from 8:00 a.m. to 2:30 p.m. and reluctantly conducted reagent loading, calibration and the instruments' maintenance from 2:30 p.m. to 5:30 p.m. It was labor-consuming and time-costing to load reagents for $3 \mathrm{~h}$ in Pattern 1. Most importantly, the clinical process was interrupted by reagent loading. These time should have been conducted another more tests instead of leaving it until tomorrow. Top 10 clinical chemistry tests of Pattern 1 were listed (Supplemental Figure 2). The average on-line time of these 


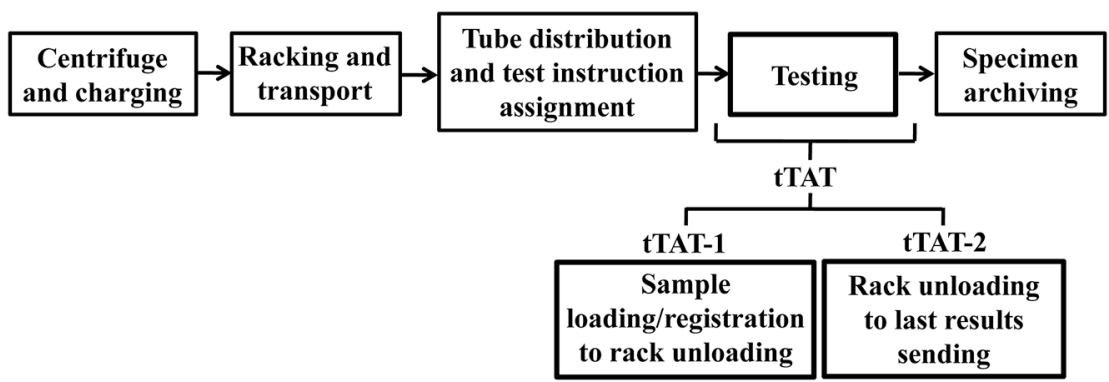

Figure 2: The workflow in our clinical chemistry laboratory and the flow dissections of tTAT. reagents was $7.6 \pm 4.39$ days (Table 2), which is a problem for reagents with short on-board stability, such as ALP, which remains stable only for 7 days at $2-8^{\circ} \mathrm{C}$. The QC cost for Pattern 1 was 35.75 Chinese Yuan. Test item performance verification times for one modular analyzer series in our lab were conducted by reagent disks. The experimental verification of Pattern 1 needs at least three times for one testing item and this was very complicated in practice.

\section{Two theoretical loading modes}

Before implementing the reagent-loading project, we conducted theoretical simulations on Pattern 2 (Supplemental Table 3) and Pattern 3 (Supplemental Table 4) based on the practical Pattern 1 data. We aim to optimize our reagentloading project to shorten the TTAT and expenses. Besides, the simulations made the best effort to reduce the reagent packs for every c701 test item, as well as the costs of performance verification and labor. All of these optimization designs were conducted as a prerequisite of at least maintaining the available efficiency of Pattern 1. Thus, TAT simulation results show that Pattern 2 would be the best choice for our lab (Table 2). Furthermore, other parameters in Table 2 reveal that Pattern 2 could be put into practice.

Table 2: The simulation results for Pattern 1, Pattern 2 and Pattern 3.

\begin{tabular}{lrrr}
\hline & Pattern 1 & Pattern 2 & Pattern 3 \\
\hline tTAT-1, tTAT (s) & $2,591 \pm 733$, & $2,473 \pm 1,097$, & $2,918 \pm 1,073$, \\
& $2,954 \pm 729$ & $2,869 \pm 983$ & $3,311 \pm 1,060$ \\
Parallel bins & 51 & 22 & None \\
$\begin{array}{l}\text { Average on- } \\
\text { line time, } \\
\text { days }\end{array}$ & $7.60 \pm 4.39$ & $4.38 \pm 2.80$ & $2.44 \pm 1.23$ \\
$\begin{array}{l}\text { QC cost, \% } \\
\begin{array}{l}\text { Performance } \\
\text { verification } \\
\text { times }\end{array} \\
\text { Labor level }\end{array}$ & 100 & $56.82 \pm 8.39$ & $35.98 \pm 13.46$ \\
\hline
\end{tabular}

\section{Pattern 2 verification}

tTAT and tTAT- 1 for Pattern 2 and Pattern 3 in Table 2 were theoretical basis calculated from the cobas 8000 simulator. The actual tTAT for Pattern 2 should be tested after optimization mode being confirmed. Before the transformation of reagent-loading manner from Pattern 1 to Pattern 2, we trained all of our technicians to make sure that all the reagents can be loaded in Pattern 2. Then we ran data in Pattern 2 under the same conditions as Pattern 1 according to the Tuesday effect. tTAT and tTAT-1 were 1,020 \pm 202 and $1,502 \pm 234 \mathrm{~s}$, respectively. In practice, tTAT and tTAT- 1 of Pattern 2 reduced 61 and 49\% than that of Pattern 1. It means that clinical chemistry results can be sent more rapidly and improve patients' satisfaction. Reagent on-line time in Pattern 1 decreased 43\% in total. This ensures the robustness of the results effectively. Furthermore, QC cost and performance verification times save the expenditures for $43 \%$. The lower labor elevated the laboratory efficiency and improved employees' satisfaction. In conclusion, Pattern 2 gets better effects.

\section{Discussion}

Aiming to shorten TAT and reduce cost, optimization on reagent-loading manner is a new breakthrough for clinical chemistry analyzer. How to define different reagentloading manners and analyze the present reagentloading data is a tangle problem. Abundant experiences and clinical chemistry tests here in our lab reminder us that simulation is the best choice for the problem. In order to get high throughput tests, two cobas 8000 c701 modules connected in parallel meet our demands. This analyzer has double simple probes and double reagent bins for every c701 module. Thus, these are three reagent-loading manners, Pattern 1, Pattern 2 and Pattern 3 for one clinical chemistry test. As the reagent-loading manner confirmed, the only remained problem is to set rules or comparative parameters which include tTAT, parallel bin, on-line time, QC cost, labor or 
work hard level and performance verification times. Based on these rules, the comparison of performance parameters for three reagent-loading manners can be carried out.

Solid theory foundation and simulation analysis has been established. Then, the last vital precondition is to make sure that the simulation can be verified and put into effect. It is impossible to complete transplant test specimen conditions of analyzer series from one day to another for the purpose of simulation verification. But, specimen rhythm of every hospital, like here the Tuesday effect in our hospital, proves that the clinical laboratory processes represented by analytical phase are same and the preanalytical phase, covered from test ordering, formulation and input of samples, sample collection to sample transportation, has been standardized. The Tuesday effect could offer credible opportunity for simulation verification and guarantee the stability and repeatability of specimen test data.

As the development of modern medicine, the patients and physicians demand faster and more accurate test items to aid for diagnosis and treatment. So, the lab executives prefer to equip the hospital with more test items in current instruments' setups. It is nearly impossible for manufacturers of clinical chemistry analyzer series to produce reagents need by all the test items on the equipment. This is the reason why user-defined reagents exist in most clinical chemistry labs. Part of user-defined reagents used in our lab provides clues about reagent-loading manner for us before simulation.

\section{Conclusions}

Simulation analysis based on current reagent-loading data differentiates three reagent-loading manners and provides the best plan for verification. Optimizations of reagentloading manner in clinical chemistry may largely improve the testing process with properer tTAT and lower cost.

Acknowledgments: The authors thank Huibo Xie, Xiaodan Gao and Xiaoming Duan technicians from Roche Dignostic for assistance in cobas 8000 simulator usages and advices. Research funding: This work was supported by 2019 Medical Science and Technology Research Project of Henan Province (No. SB201902009). The funding organization(s) played no role in the study design; in the collection, analysis, and interpretation of data; in the writing of the report; or in the decision to submit the report for publication.
Author contributions: All authors have accepted responsibility for the entire content of this manuscript and approved its submission.

Competing interests: Authors state no conflict of interest. Ethical approval: The local Institutional Review Board deemed the study exempt from review.

\section{References}

1. Mcpherson RA. Automation in the clinical laboratory. In: Encyclopedia of analytical chemistry. Hoboken, NJ: John Wiley \& Sons Ltd; 2006.

2. Nada M-S, Zorica Š. Quality indicators of the pre-analytical phase. J Med Biochem 2012;31:174-83.

3. Lou AH, Elnenaei MO, Sadek I, Thompson S, Crocker BD, Nassar BA. Multiple pre- and post-analytical lean approaches to the improvement of the laboratory turnaround time in a large core laboratory. Clin Bio Chem 2017;04:019.

4. Sarkozi L, Simson E, Ramanathan L. The effects of total laboratory automation on the management of a clinical chemistry laboratory. Retrospective analysis of 36 years. Clin Chim Acta 2003;329: 89-94.

5. Hawker CD, Roberts WL, Garr SB, Hamilton LT, Penrose JR, Ashwood ER, et al. Automated transport and sorting system in a large reference laboratory: part 2. Implementation of the system and performance measures over three years. Clin Chem 2002;48: 1761-7.

6. Chung H-J, Song Y K, Hwang S-H, Lee DH, Sugiura T. Experimental fusion of different versions of the total laboratory automation system and improvement of laboratory turnaround time. J Clin Lab Anal 2018;32:e22400.

7. Lam CW, Jacob E. Implementing a laboratory automation system: experience of a large clinical laboratory. J Lab Autom 2012;17:16-23.

8. Ialongo C, Porzio O, Giambini I, Bernardini S. Total automation for the core laboratory: improving the turnaround time helps to reduce the volume of ordered STAT tests. J Lab Autom 2016;21:451.

9. Wahed MA, Ahmed NK, Wahba KK. Reducing laboratory total turnaround time (TAT) using system dynamics simulation: chemistry analyzer application. The 7th Cairo International Biomedical Engineering Conference (CIBEC 2014). Cairo: IEEE; 2014: pp.99-102.

10. Nader R. Tietz textbook of clinical chemistry and molecular diagnostics, 6th ed. St. Louis, Missouri: Elsevier; 2018:370 p.

11. Roundy CS, Lin DC, Klopping PJ, Ence AT, Krezel AC, Genzen JR. Specimen temperature detection on a clinical laboratory preanalytic automation track: implications for direct-from-Track Total Laboratory Automation (TLA) systems. SLAS Technol 2019; 25:1-7.

12. Hubl W, Zogbaum M, Boyd JC, Savory J, Schubert M, Meyer D, et al. Evaluation of analytical methods and workflow performance of the Architect ci8200 integrated serum/plasma analyzer system. Clin Chim Acta 2005;357:0-54.

Supplementary Material: The online version of this article offers supplementary material (https://doi.org/10.1515/labmed-2020-0075). 